\title{
H.264/MPEG-4 AVC Video Streaming Analysis of AODV, OLSR and ZRP in MANET
}

Dr.P.Periyasamy

Department of Computer Science and Applications, Sree Saraswathi Thyagaraja College, Pollachi - 642 107, Tamil

Nadu, India

Email: pereee@yahoo.com

Conversational real-time applications like VoIP (Voice over IP) and videoconferencing require new challenges in Long-Term Evolution (LTE) networks such as Internet. Quality of video delivered over the Internet has been determined in terms of Quality-of-Service (QoS) and Quality-of-Experience (QoE). QoS includes packet delivery ratio, average end-to-end delay, throughput, routing overhead, etc. QoE is a qualitative measure of videos transmitted over Internet. In this paper, the performance comparison of AODV, OLSR and ZRP are carried out on H.264/MPEG-4 AVC video streaming under both Distributed Coordination Function (DCF) and Enhanced Distributed Coordination Function (EDCF) using NS2.34 and Enhanced EvalVid framework. These protocols have been selected for simulation due to their edges over alternative protocols in numerous aspects.

Keywords - MANET, QoS, QoE, VoIP, AODV, OLSR, ZRP, LTE, DCF, EDCF.

\section{INTRODUCTION}

Mobile Ad hoc NETwork (MANET) is a collection of mobile nodes forming a dynamic topology by wireless links without any network infrastructure such as routers, servers, access points/cables or centralized administration. Each mobile node is performing as a router as well as a node. The most important characteristics of MANET are i) Dynamic topologies ii) Bandwidth constrained links iii) Energy constrained operation and iv) limited physical security [1,2]. Routing protocols play a vital role in MANET to find routes for packet delivery and make sure that the packets are delivered to the correct destinations. These protocols are classified as: (i) proactive - find routes proactively based on the routing table (i.e., table-driven), (ii) reactive - find routes whenever needed (i.e., ondemand), and (iii) hybrid - the combination of both proactive and reactive.

In this paper, the performance comparison of Ad Hoc Ondemand Distance Vector (AODV), Optimized Link State Routing (OLSR) and Zone Routing Protocol (ZRP) is carried out on H.264/MPEG-4 AVC video streaming under both Distributed Coordination Function (DCF) and Enhanced Distributed Coordination Function (EDCF) using NS2.34 and Enhanced EvalVid framework. These protocols have been selected for simulation due to their edges over alternative protocols in numerous aspects.

The rest of the paper is organized as follows: Section II presents the overview of AODV, OLSR, and ZRP. In Section III, the overview of EvalVid framework is presented. In Section IV, the Video Quality Metrics such as Quality-of-Service (QoS) and Quality-of-Experience (QoE) are given. The simulation and experimental results are discussed in Section V. The conclusions and future work are given in Section VI.

\section{OVERVIEW OF AODV, OLSR AND ZRP}

The communication within the network is facilitated through a protocol which establishes the correct and efficient route between a pair of nodes so that messages may be delivered in a timely manner. The primary characteristics of a protocol are to establish routes with a minimum of overheads and bandwidth consumptions.

\subsection{Ad Hoc On-demand Distance Vector (AODV)}

The AODV [3] protocol is a simple and widely used ondemand unipath routing protocol that starts a route discovery process through a route request (RREQ) to the destination throughout the network when needed for MANE. Once a non-duplicate RREQ is received, the intermediate node records the previous hop and checks for a valid and fresh route entry to the destination. The node sends a route reply (RREP) with a unique sequence number to the source. On updating the route information, it propagates the route reply and gets additional RREPs if a RREP has either a larger destination sequence number (fresher) or a shorter route found.

\subsection{Optimized Link State Routing (OLSR)}

The OLSR $[4,5,6]$ protocol is an optimization of a pure link state protocol by compacting the size of the control packets that contain link-state information and reducing the number of transmissions by flooding those control packets to the entire network. The multipoint relaying technique helps to flood its control messages in an efficient and economic way. The main aim of multipoint relays is to minimize the flooding of broadcast packets in the network by reducing the number of retransmissions in the same region. Each node selects a set of 1- hop neighbour nodes, called the multipoint relays (MPRs) of that node, which retransmits its packets in OLSR. The neighbours of any node $\mathrm{N}$ do not retransmit the broadcast 
packets received from node $\mathrm{N}$ if they are not in the MPR set whereas they can read and process packets. Each node have a set of neighbours for retransmission of packets called MPR Selectors.

\subsection{Zone Routing Protocol (ZRP)}

The ZRP [7,8,9] combines the advantages of proactive and reactive protocols in a hybrid scheme. It acts as a proactive protocol in the neighbourhood of a node (IntrA-zone Routing Protocol, IARP) locally and a reactive protocol for routing between neighbourhoods (IntEr-zone Routing Protocol, IERP) globally. The local neighbourhoods are called zones, which are different for each node. Each node may be within multiple overlapping zones and each zone may be of a different size. The "size" of a zone isn't determined by the geographical mensuration however is set by a radius of length.

\section{OVERVIEW OF ENHANCED EVALVID FRAMEWORK}

\subsection{MPEG -4 Video Coding Terminology}

MPEG-4 [10] video Sequence comprises of three types of frames such as (i) I-frames (Intra-coded) - Frames in a video clip that are compressed using the combination of various lossless and lossy compression techniques without making reference to any previous or subsequent frame in the sequence. These frames are the largest of the three with highest-quality but they are the least efficient from a compression perspective. These are also known as keyframes. (ii) P-frames (predicted) - Frames in a video clip that are produced by the encoder using a backward reference to previous I or P-frames for redundant picture information. P-frames are more efficient than I-frames but less efficient than B-frames is shown in Figure 1. (iii) Bframes (Bi-directional) - Frames in a video clip that are produced by the encoder using both forward and backward reference to previous/next I or P-frames for redundant picture information. B-frames are the most efficient frame of the three but they are not available in H.264's Baseline Profile is shown in Figure 2.

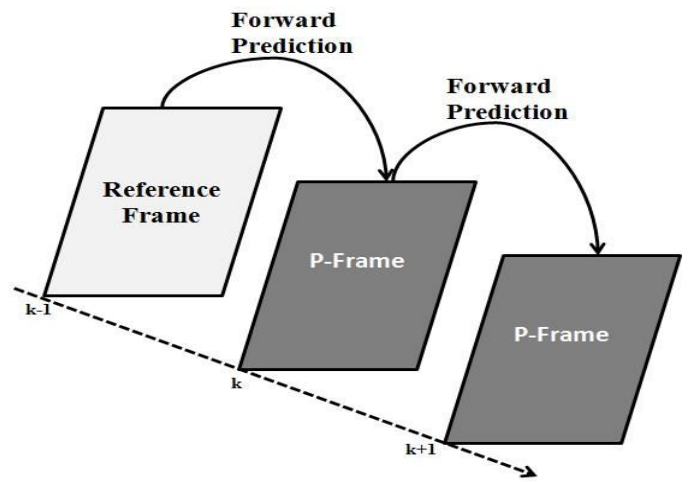

Figure 1. Concept of a P-Frame [12]

Most broadcast qualitative applications have a propensity to use two consecutive $\mathrm{B}$ frames $(\mathrm{I}, \mathrm{B}, \mathrm{B}, \mathrm{P}, \mathrm{B}, \mathrm{B}, \mathrm{P}, \ldots)$ for the ideal trade-off between compression efficiency and video quality. Figure 3 shows an example of MPEG video frame sequence. Quarter Common Intermediate Format (QCIF) is selected because of its acceptable resolution efficiency and suitability in bit-rate and frame rate by providing 56 kbps and $30 \mathrm{fps}$ (frames per second) respectively.

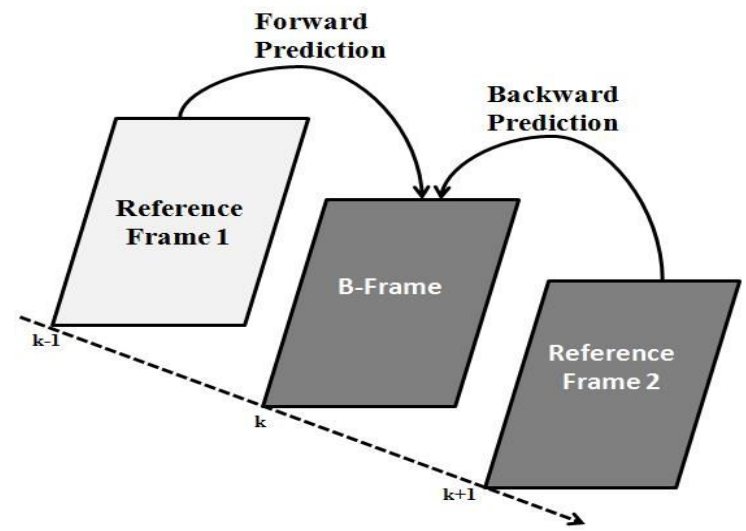

Figure 2. Concept of a B-Frame [12]

The main advantage of $\mathrm{B}$ frames is to enhance coding efficiency. The B frames will result in fewer bits being coded overall in many cases and the quality can also be improved in the case of moving objects that reveal the hidden areas within a video sequence. Backward prediction allows the encoder to make more intelligent decisions on how to encode the video within these areas in this case. Since B frames are not used to predict future frames, the generated errors will not be propagated further within the sequence.

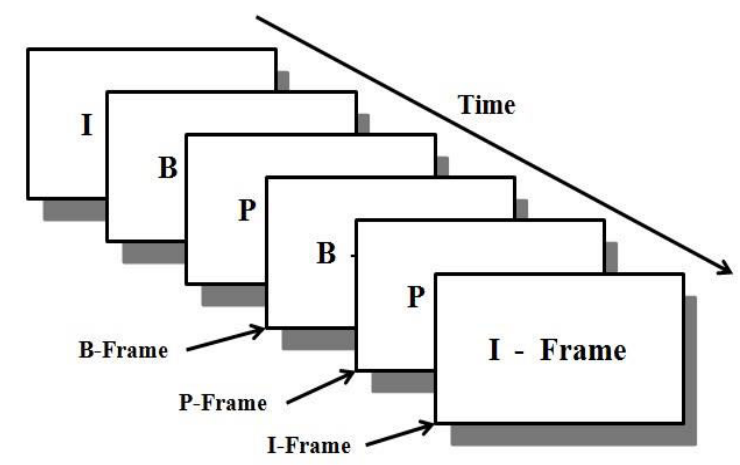

Figure 3. MPEG Video Frame Types [12]

\subsection{Enhanced EvalVid framework}

Enhanced EvalVid [11,12] is an extended framework and tool-set of EvalVid [13,14] for evaluating the quality of the H.264/MPEG-4 AVC video transmitted over a real or simulated communication network. This framework provides a packet/frame loss rate, packet/frame jitter, PSNR, and MOS metrics for video quality assessment purposes. The structure of the enhanced EvalVid framework is shown in Figure 4. The main components of the enhanced EvalVid are described as follows:

(i) Source - It can be a video either in the YUV QCIF (176 $\times 144)$ or in the YUV CIF $(352 \times 288)$ formats.

(ii) Video Encoder and Video Decoder - The enhanced EvalVid supports three kinds of MPEG4 codecs such as 
NCTU codec [15], FFmpeg [16] and Xvid [17] but the original EvalVid supports only a single layer video coding. The focus of this investigation is NCTU codec for video committal to writing functions.

(iii) VS (Video Sender) - This component is used to read the compressed video file from the output of the video encoder, each large video frame is fragmented into smaller segments, and then transmitted these segments via UDP packets over a real or simulated network. This framework records the timestamp, the packet ID, and the packet payload size of each transmitted UDP packet in the sender trace file with the help of third-party tools such as tcpdump [18] or win-dump [19] if the network is a real link.

(iv) ET (Evaluate Trace) - Once the video transmission is over, the data regarding the timestamp, the packet ID and also the packet payload size out there at the receiver has got to be transported back to the sender in order to begin the evaluation task at the sender side. The ET part creates a frame/packet loss and frame/packet disturbance report and generates a reconstructed video file supported the initial encoded video file, the video trace file, the sender trace file and the receiver trace file which corresponds to the possibly corrupted video found at the receiver side as it would be reproduced to an end user.

(v) FV (Fix Video) - Digital video quality assessment is performed frame by frame. Therefore, the total number of video frames at the receiver side including the erroneous frames must be the same as that of the original video at the sender side. If the codec cannot handle missing frames, the FV part is employed to tackle this drawback by inserting the last with success decoded frame the place of every lost frame as an error concealment technique [20].

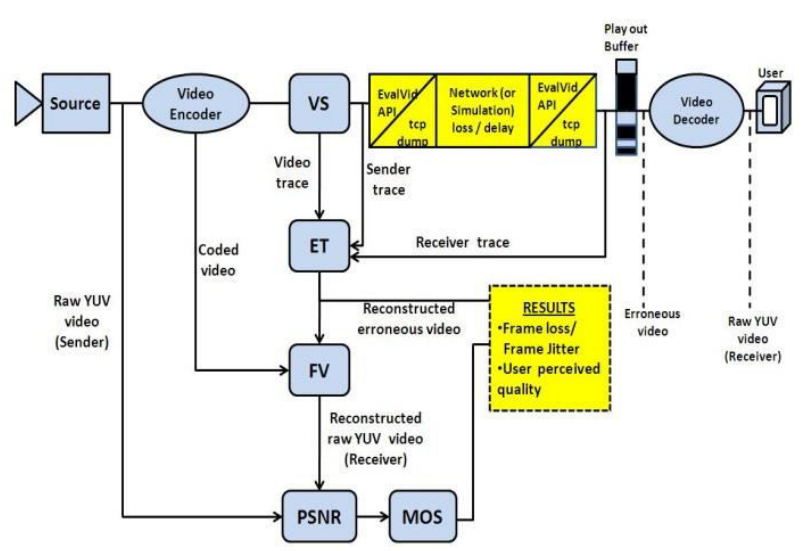

Figure 4. Structure of enhanced EvalVid framework $[11,12]$

\subsection{Integrated structure of enhanced EvalVid framework with NS2}

Figure 5. illustrates the integrated structure of enhanced EvalVid with NS2. The enhanced EvalVid has the following three interface programs for communicating with NS2: MyTrafficTrace, My_UDP, and MyEvalvid_Sink:

(i) MyTrafficTrace [11,12] agent extracts the frame type and the frame size of the video trace file generated from the traffic trace file, fragments the video frames into smaller segments and sends these segments to the lower UDP layer at the acceptable time in step with the user settings per the simulation script file.

(ii) My_UDP [11,12] is an extension of the UDP agent that allows the user to specify the output file that is the name of the sender trace file which records the timestamp of every transmitted packet, the packet ID, and the packet payload size. The task of the My_UDP agent corresponds to the task that tools such as tcp-dump or win-dump performs in a real network environment.

(iii) MyEvalvid_Sink [11,12] is the receiving agent for the fragmented video frame packets sent by My_UDP. This agent also records the timestamp, packet ID, and payload size of each received packet which have been kept in the user specified receiver trace file.

As a result, the total analysis method starts with coding the raw YUV video, then the VS program can scan the compressed file and generate the traffic trace file.

After the simulation, the ET program produces the corrupted video file based on these three trace files and the original encoded video. Subsequently, the corrupted video is decoded and error concealed. Finally, the reconstructed fixed YUV video can be compared with the original raw YUV video to evaluate the end-to-end delivered video quality.

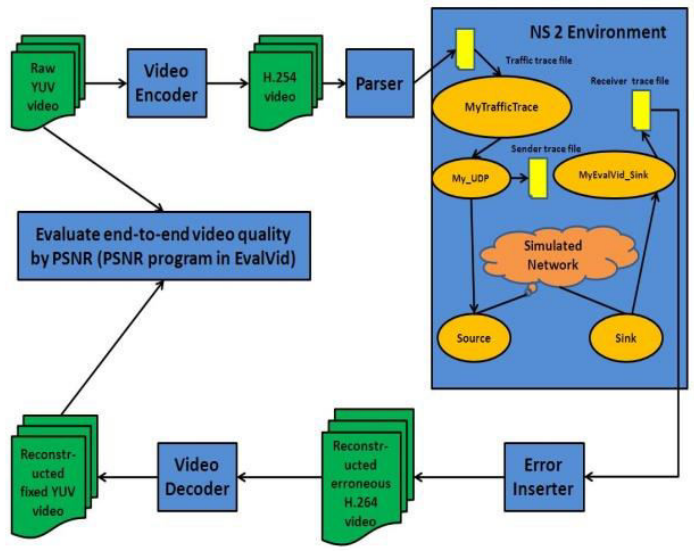

Figure 5. Integrated structure of the enhanced EvalVid with ns-2 [11, 12]

\section{VIDEO QUALITY METRICS}

Quality of Service $(Q \circ S)$ and Quality of Experience $(Q o S)$ are the qualitative measures of the videos have to be delivered over wireless communication networks but QoE reflects the user perception. The most widely used QoE metrics are as follows: 


\subsection{Peak Signal-to-Noise Ratio (PSNR)}

Peak Signal-to-Noise Ratio (PSNR) $[11,12]$ is a traditional objective quality metric used to measure the video quality level based on original and processed video sequences. PSNR of the frames with $\mathrm{M} \times \mathrm{N}$ pixels and 8 bits/sample is defined using Equation 1 as follows:

$$
P S N R=20 \log _{10}\left[\frac{255}{\sqrt{\frac{1}{M x N} \sum_{i=0}^{M-1 N-1} \sum_{j=0}^{N-1}\|Y(i . j)-Y d(i, j)\|^{2}}}\right]
$$

\subsection{Mean Opinion Score (MOS)}

The most widely used subjective quality measure which measures the quality of a system by using "opinion score" in ITU-T Recommendation P.800 is called Mean Opinion Score (MOS) [12, 21, 22]. Depending on the quality factors to be assessed, MOS can be classified into assessments of listening quality and conversation quality. Possible PSNR to MOS conversion under Absolute Category Rating (ACR) [22] test is shown in Table 1. MOS can be assessed in terms of listening quality and conversation quality. The listening quality expresses the quality experienced by users when listening to speech and the conversation quality expresses the quality experienced by users when taking part in a conversation.

Table 1. Possible PSNR to MOS conversion under Absolute Category Rating (ACR) test

\begin{tabular}{|l|l|l|}
\hline PSNR [dB] & MOS & Category \\
\hline$>37$ & 5 & Excellent \\
\hline $31-37$ & 4 & Good \\
\hline $25-31$ & 3 & Fair \\
\hline $20-25$ & 2 & Poor \\
\hline$<20$ & 1 & Bad \\
\hline
\end{tabular}

\subsection{Throughput (in Kbps)}

Throughput (in Kbps) is the number of bytes received successfully and is calculated as follows:

$$
\text { Throughput }=\frac{\text { No. of Bytes } \text { Received } * 8}{\text { Simulation Time } * 1000} \text { kbs }
$$

\subsection{Average End-to-End delay (in $\mathrm{ms}$ )}

Average End-to-End delay (in $\mathrm{ms}$ ) is the average time of the data packet to be successfully transmitted across a MANET from source to destination. It includes all attainable delays like buffering throughout the route discovery latency, queuing at the interface queue, retransmission delay at the MAC, the propagation and the transfer time and is calculated as follows:
Average End - to - End delay $=\frac{\sum_{i=1}^{n}\left(R_{i}-S_{i}\right)}{\mathrm{n}} m s$

Where $\mathrm{n}$ is the number of data packets successfully transmitted over the MANET, ' $i$ ' is the unique packet identifier, $R_{i}$ is the time at which a packet with unique identifier ' $\mathrm{i}$ ' is received and $\mathrm{S}_{\mathrm{i}}$ is the time at which a packet with unique identifier ' $i$ ' is sent. The Average End-to-End Delay ought to be less for top performance.

\section{EXPERIMENTAL RESULTS AND DISCUSSION}

The performance comparison of Ad Hoc On-demand Distance Vector (AODV), Optimized Link State Routing (OLSR) and Zone Routing Protocol (ZRP) are carried out on H.264/MPEG-4 AVC video streaming under both Distributed Coordination Function (DCF) and Enhanced Distributed Coordination Function (EDCF) using NS2.34 [23,24,25,26,27] and Enhanced EvalVid framework $[11,12]$. Table 2 shows the simulation parameters. Quarter Common Intermediate Format (QCIF) is selected for video streaming because it provides an acceptable resolution efficiency and suitability in bit-rate and frame rate by providing $56 \mathrm{kbps}$ and $30 \mathrm{fps}$ (frames per second) respectively.

Table 2. Simulation parameters

\begin{tabular}{|l|l|}
\hline Parameter (s) & Value (s) \\
\hline Simulator & NS-2.34 \\
\hline MAC Types & 802.11 DCF \& EDCF \\
\hline Simulation Area & $400 \mathrm{~m}$ x 500 m \\
\hline Simulation Time & 50 seconds \\
\hline Channel Type & Wireless Channel \\
\hline Routing Protocols & AODV, OLSR and ZRP \\
\hline Antenna Model & Omni \\
\hline Radio Propagation Model & TwoRayGround \\
\hline Packet size & 2 Kbyte \\
\hline Hello packet size & $1 \mathrm{Kbyte}$ \\
\hline Other control packet size & 100 Byte \\
\hline Frame size & 176 x 144 (QCIF) \\
\hline Bits per pixel & 0.2 \\
\hline Bit rate & $56 \mathrm{kbps}$ \\
\hline Maximum tolerable delay & 250 ms \\
\hline Average codec power/packet & $500 \mathrm{~mW}$ \\
\hline Average compression delay & $50-60 \mathrm{~ms}$ \\
\hline
\end{tabular}




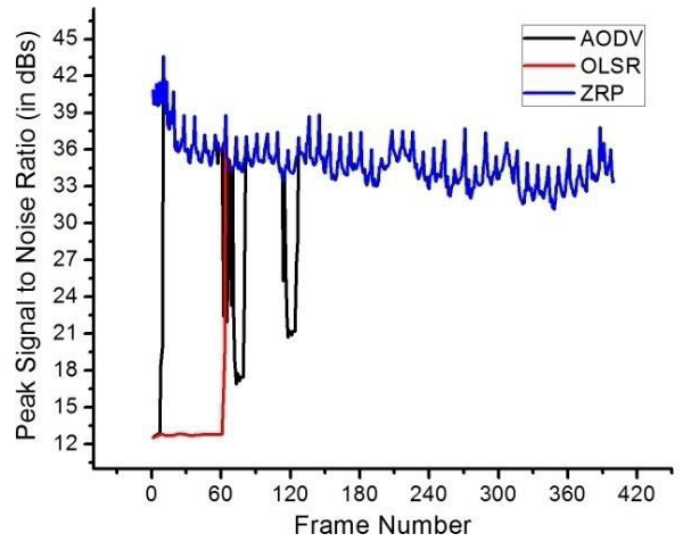

Figure 6. Peak Signal to Noise Ratio (dB) under DCA

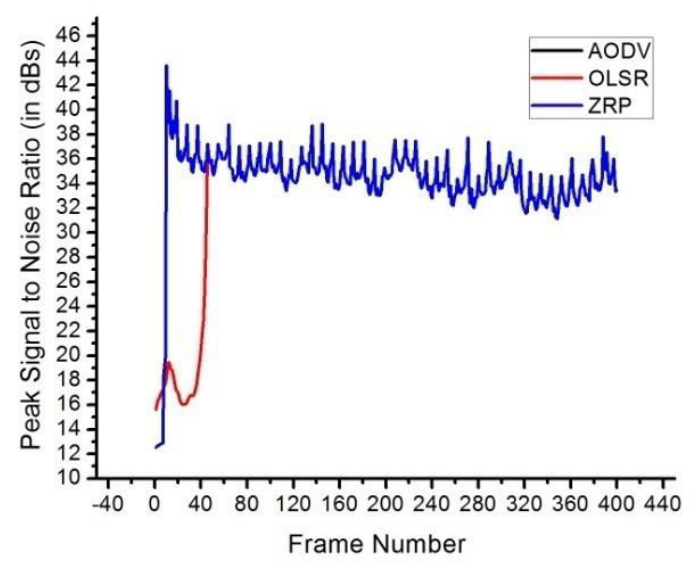

Figure 7. Peak Signal to Noise Ratio (dB) under EDCA

From Figure 6 and Figure 7, it is found that the Peak Signal to Noise Ratio (dB) of ZRP is better than the Peak Signal to Noise Ratio (dB) of AODV and OLSR under both DCA and EDCA.

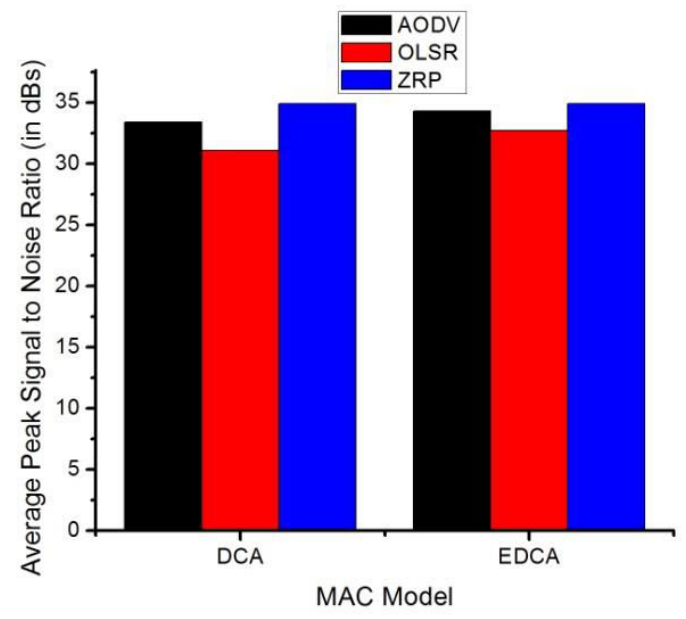

Figure 8. Average PSNR (in dBs)

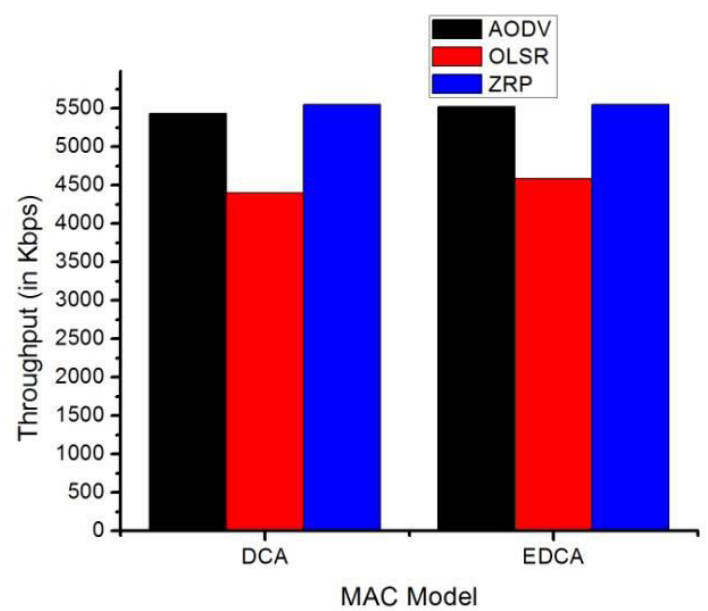

Figure 9. Average Throughput (in bps)

From Figure 8 and Figure 9, it is also found that the Average PSNR and Throughput of ZRP is better than the Average PSNR and Throughput of AODV and OLSR under both DCA and EDCA.

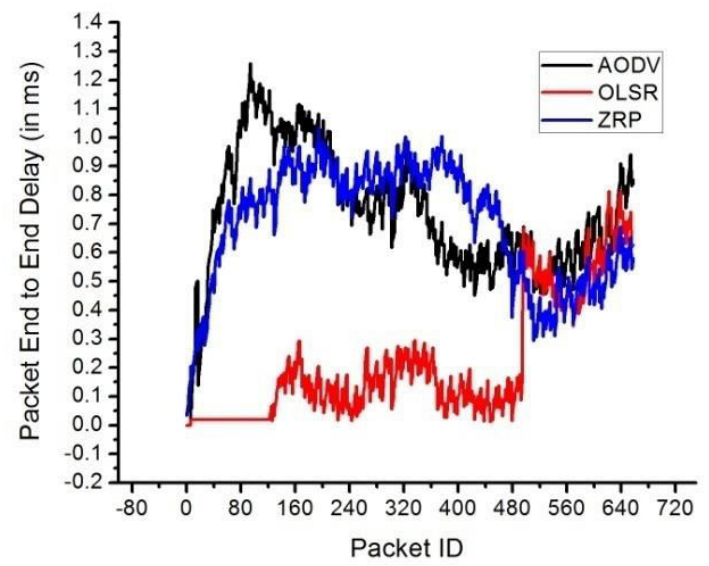

Figure 10. Packet End-to-End Delay (in ms) in DCA

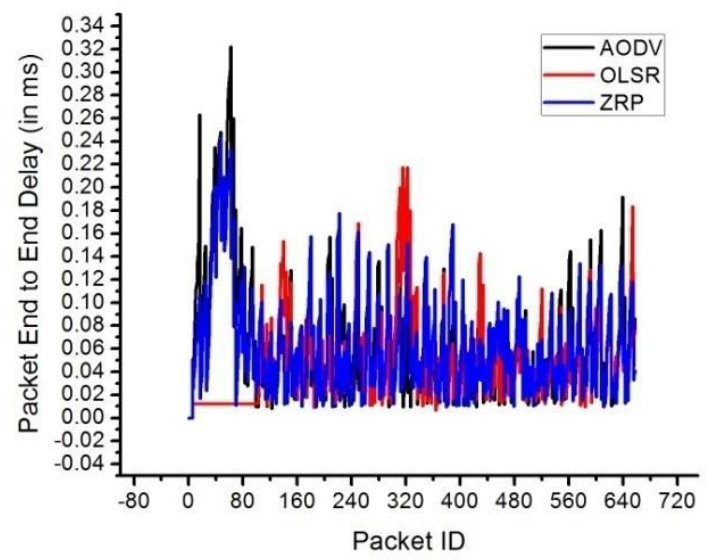

Figure 11. Packet End-to-End Delay (in ms) in EDCA 


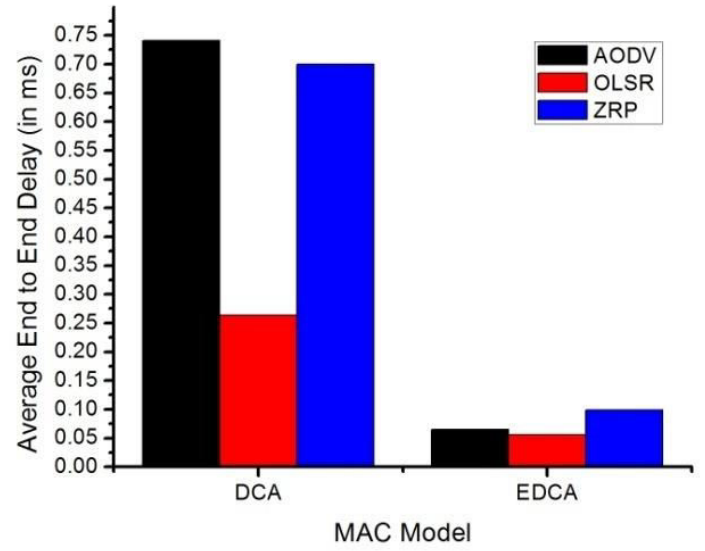

Figure 12. Average End-to-End Delay (in ms)

From Figure 10, Figure 11 and Figure 12, it is found that the Packet End-to-End Delay and Average End-to-End Delay of OLSR is better than the Packet End-to-End Delay and Average End-to-End Delay of AODV and ZRP under both DCA and EDCA.

\section{CONCLUSION AND FUTURE WORK}

Conversational real-time applications like VoIP (Voice over IP) and videoconferencing require new challenges in Long-Term Evolution (LTE) networks such as Internet. Quality of video delivered over the Internet has been determined in terms of Quality-of-Service (QoS) and Quality-of-Experience (QoE). QoS includes packet delivery ratio, average end-to-end delay, throughput, routing overhead, etc. QoE is a qualitative measure of videos transmitted over Internet. The performance of AODV, OLSR and ZRP are investigated on H.264/MPEG4 AVC video streaming under both Distributed Coordination Function (DCF) and Enhanced Distributed Coordination Function (EDCF) using NS2.34 and Enhanced EvalVid framework. These protocols are chosen for simulation thanks to their edges over alternative protocols in numerous aspects. Packet End-to-End Delay (in ms) and Average End-to-End Delay (in ms) of OLSR is better than the Packet End-to-End Delay (in ms) and Average End-to-End Delay (in ms) of AODV and ZRP. Average PSNR and Throughput of ZRP is better than the Average PSNR and Throughput of AODV and OLSR under both DCA and EDCA. In future, video streaming analysis of AODV, OLSR and ZRP will be carried out in cross layer design environment. This evaluation will also be carried out by applying Scalable Video Coding (SVC) techniques.

\section{REFERENCES}

[1] Sapna S. Kaushik, P.R.Deshmukh, "Comparison of effectiveness of AODV, DSDV and DSR Routing Protocols in Mobile Ad hoc Networks", International Journal of Information Technology and Knowledge Management, Vol. 2(2), pp.499-502, 2009.
[2] V.C.Patil, Rajashree, V.Biradar, R.R. Mudholkar, S.R.Sawant, "On-Demand Multipath Routing Protocols for Mobile Ad Hoc Networks Issues and Comparison", International Journal of Wireless Communication and Simulation, Vol. 2(1), pp. 2138, 2010.

[3] C.E.Perkins and E.M.Royer, "Ad hoc on-demand distance vector routing”, Proc. IEEE Workshop on Mobile Computing Systems and Applications, New Orleans, LA, pp. 90-100, 1999.

[4] T. Clausen, P. Jacquet, A. Laouiti, P. Muhlethaler, A. Qayyum and L. Viennot, "Optimized Link State Routing Protocol for Mobile Ad Hoc Networks", IEEE INMIC, Pakistan 2001.

[5] P. Jacquet, P. Muhlethaler, and A. Qayyum, "Optimized Link State Routing Protocol", IETF Internet Draft, draft-ietf-manet-olsr-10.txt, June 2002.

[6] P. Jacquet and T. Clausen, "Optimized Link State Routing Protocol", IETF Internet Draft, draftietfmanet-olsr-11.txt, July 2003.

[7] J. Schaumann, "Analysis of the Zone Routing Protocol”, December 2002.

[8] Z. Haas and M. Pearlman, "The zone routing protocol (ZRP) for Ad Hoc networks", IETF Internet Draft, draft-ietf-manet-zone-zrp-04.txt, July 2002.

[9] Z. Haas, "A New Routing Protocol for the Reconfigurable Wireless Networks", Proceedings of IEEE ICUPC'97, San Diego, CA, pp. 562-566, October 1997.

[10] J. Ostermann, J. Bormans, P. List, D. Marpe, M. Narroschke, F. Pereira, T. Stockhammer, and T. Wedi. "Video coding with H.264/AVC: tools, performance, and complexity", Circuits and Systems Magazine, IEEE, Vol.4, No.1, pp. 7-28, Feb 2004.

[11] C. H. Ke, C. K. Shieh, W. S. Hwang, A. Ziviani, “An Evaluation Framework for More Realistic Simulations of MPEG Video Transmission", Journal of Information Science and Engineering, Vol.24, No.2, pp.425-440, March 2008.

[12] P.Periyasamy and E.Karthikeyan, "H.264/MPEG-4 AVC Video Streaming Evaluation of LR-EEAOMDV protocol in MANET", CIT. Journal of Computing and Information Technology, Vol. 25, No. 1, pp. 15-29, 2017.

[13] J. Klaue, B. Rathke, and A. Wolisz, "EvalVid - A framework for video transmission and quality evaluation", Proc. of the International Conference on 
Modelling Techniques and Tools for Computer Performance Evaluation, pp. 255-272, 2003.

[14] Enhanced EvalVid Framework: http://hpds.ee.ncku.edu.tw/ smallko/ns2/Evalvid_in_ NS2.htm.

[15] NCTU codec, http://megaera.ee.nctu.edu.tw/mpeg.

[16] ffmpeg, http://ffmpeg.sourceforge.net/index.php.

[17] Xvid, http://www.xvid.org/.

[18] tcp-dump, http://www.tcpdump.org.

[19] win-dump, http://windump.polito.it.

[20] Y. Wang and Q. F. Zhu, "Error control and concealment for video communication: a review", Proceedings of the IEEE, Vol. 86, 1998, pp. 974-997.

[21] Speech quality assessment methods, http://www.ntt.co.jp/qos/qoe/eng/technology/sound/i ndex.html

[22] Video quality assessment methods, http://www.ntt.co.jp/qos/qoe/eng/technology/visual/i $\underline{\text { ndex.html }}$

[23] The Network Simulator ns-allinone-2.34, http://www.isi.edu/nsnam/ns/

[24] Kevin Fall, K. Varadhan, "The ns Manual", University of Southern California, Information Sciences Institute (ISI), http://www.isi.edu/nsnam/ns/ns-documentation.html.

[25] NS-2 with Wireless and Mobility Extensions, http://monarch.cs.rice.edu/cmu-ns.html

[26] P.Periyasamy and E.Karthikeyan, "A simulation based QoS review of multipath routing protocols for MANET", International Journal of Advanced Networking and Applications (IJANA), Vol.4, No.3, pp. 1624-1634, 2012.

[27] P.Periyasamy and E.Karthikeyan, "Link Reliable Joint Path and Spectrum Diversity in Cognitive Radio Ad-Hoc Networks", International Journal of Advanced Networking and Applications (IJANA), Vol.8, No.2, pp. 3036-3043, 2016.

\section{Author Biography}

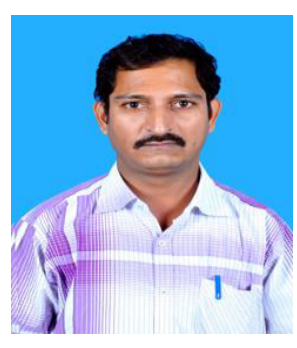

Dr. P. Periyasamy is an Associate Professor in Computer Science and Applications at Sree Saraswathi Thyagaraja College, India. His research interests include the design and development of routing protocols to enhance the quality of service (QoS) and quality of experience (QoE) requirements for wireless networks such as MANETs, VANETs, CRAHNs and DTNs. He has published 18 papers in international journals and 2 conference proceedings. He has attended more than 10 conferences at national and international levels. He is a reviewer for the journals IEEE Access, Wireless Personal Communications, KSII Transactions on Internet and Information Systems, Journal of Communications and Information Networks Journal. He is an editorial board member for the Information and Computer Security Journal. 